\title{
Analysis of the electrical behavior of silicon rich silicon oxides
}

\section{Análisis del comportamiento eléctrico de óxidos de silicio enriquecidos en silicio}

\author{
Joan Juvert $(1, *)$, Alfredo González(1), Alfredo Morales-Sánchez(2), Jorge Barreto(3), \\ Mariano Aceves-Mijares(4), Carlos Domínguez(1) \\ 1. Dept. Micro y Nanosistemas, Institut de Microelectrònica de Barcelona (IMB-CNM, CSIC), Campus UAB, 08193 \\ Bellaterra, Barcelona, Spain. \\ 2. CIDS-ICUAP, Benemérita Universidad Autónoma de Puebla, Puebla, Mexico. \\ 3. NPRL, School of Physics and Astronomy, Universtiy of Birmingham, Birmingham B15 2TT UK. \\ 4. INAOE, dpt. of Electronics, PO box 51, Puebla, Pue. 72000,México. \\ ${ }^{(*)}$ Email: joan.juvert@imb-cnm.csic.es \\ Recibido / Received: 29/09/2011. Revisado / Revised: 14/12/2011. Aceptado / Accepted: 15/12/2011.
}

\begin{abstract}
:
The electrical behavior (capacitance-voltage and current-voltage) of MOS-like structures with silicon rich silicon oxide (SRO) as the dielectric material has been studied. The SRO active layer has been obtained by three different CMOS compatible techniques, namely low pressure chemical vapor deposition (LPCVD), plasma enhanced chemical vapor deposition (PECVD) and ion implantation. Different silicon excesses have been analyzed. The results have been related to the electroluminescent behavior of the samples. Two different conduction regimes have been identified: a high leakage regime and a low leakage regime. The former is related to an anomalous $\mathrm{C}-\mathrm{V}$ behavior and to the luminescence from a limited number of dots in the area of the devices whereas the latter is related to a regular $\mathrm{C}-\mathrm{V}$ behavior and to the homogeneous luminescence of the whole area of the devices.
\end{abstract}

Key words: LPCVD, PECVD, Implantation, Silicon, Nanoparticles, Electroluminescence.

\section{RESUMEN:}

Se ha estudiado el comportamiento eléctrico (capacitancia-voltaje y corriente-voltaje) de estructuras tipo MOS con óxido de silicio enriquecido en silicio (SRO) como material dieléctrico. La capa activa de SRO ha sido obtenida por tres técnicas compatibles CMOS diferentes, como son la deposición química de vapor a baja presión (LPCVD), la deposición química de vapor asistida por plasma (PECVD) y la implantación iónica. Se han analizado diferentes excesos de silicio. Los resultados se han relacionado con el comportamiento electroluminiscente de las muestras. Se han identificado dos regímenes de conducción distintos: un régimen de altas pérdidas y un régimen de bajas pérdidas. El primero se ha asociado a un comportamiento $\mathrm{C}-\mathrm{V}$ anómalo y a luminiscencia a través de un número limitado de puntos en el área de los dispositivos, mientras que el último se ha relacionado con un comportamiento $\mathrm{C}-\mathrm{V}$ ordinario y a una luminiscencia homogénea en el área de los dispositivos.

Palabras clave: LPCVD, PECVD, Implantación, Silicio, Nanopartículas, Electroluminiscencia.

\section{REFERENCIAS Y ENLACES / REFERENCES AND LINKS}

[1]. L. T. Canham, "Silicon quantum wire array fabrication by electrochemical and chemical dissolution of wafers", Appl. Phys. Lett. 57, 1046-1048 (1990).

[2]. J. Barreto, M. Perálvarez, J. Antonio Rodríguez, A. Morales, M. Riera, M. López, B. Garrido, L. Lechuga, C. Dominguez, "Pulsed electroluminescence in silicon nanocrystals-based devices fabricated by PECVD", Physica E 38, 193-196 (2007). 
[3]. D. J. DiMaria, J. R. Kirtley, E. J. Pakulis, D. W. Dong, T. S. Kuan, F. L. Pesavento, T. N. Theis, J. A. Cutro, S. D. Brorson, "Electroluminescence studies in silicon dioxide films containing tiny silicon islands", $J$. Appl. Phys. 56, 401-416 (1984).

[4]. G. Franzò, A. Irrera, E. C. Moreira, M. Miritello, F. Iacona, D. Sanfilippo, G. Di Stefano, P. G. Fallica, F. Priolo, "Electroluminescence of silicon nanocrystals in MOS structures", Appl. Phys. A 74, 1-5, (2002).

[5]. A. Irera, D. Pacifici, M. Miritello, G. Franzò, F. Priolo, F. Iacona, D. Sanfilippo, G. Di Stefano, P. G. Fallica, "Excitation and de-excitation properties of silicon quantum dots under electrical pumping", Appl. Phys. Lett. 81, 1866-1868 (2002).

[6]. A. Morales-Sánchez, M. Aceves-Mijares, A. A. González-Fernández, K. Monfil-Leyva, J. Juvert, C. Domínguez-Horna, "Blue and red electroluminescence of silicon-rich oxide light emitting capacitors", Proc. SPIE 7719, 77190N (2010).

[7]. M. Perálvarez, C. García, M. López, B. Garrido, J. Barreto, C. Domínguez, J. A. Rodríguez, "Field effect luminescence from Si nanocrystals obtained by plasma-enhanced chemical vapor deposition", Appl. Phys. Lett. 89, 051112 (2006).

[8]. Z. H. Cen, T. P. Chen, L. Ding, Y. Liu, J. I. Wong, M. Yang, Z. Liu, W. P. Goh, F. R. Zhu, S. Fung, "Strong violet and green-yellow electroluminescence from silicon nitride thin films multiply implanted with Si ions", Appl. Phys. Lett. 94, 041102 (2009).

[9]. L. Dal Negro, J. H. Yi, J. Michel, L. C. Kimerling, T.-W. F. Chang, V. Sukhovatkin, E. H. Sargent, "Light emission efficiency and dynamics in silicon-rich silicon nitride films", Appl. Phys. Lett. 88, 233109 (2006).

[10]. R. Huang, K. Chen, H. Dong, D. Wang, H. Ding, W. Li, J. Xu, Z. Ma, L. Xu, "Enhanced electroluminescence efficiency of oxidized amorphous silicon nitride light emitting devices by modulating Si/N ratio", Appl. Phys. Lett. 91, 111104 (2007).

[11]. D. Li, J. Huang, D. Yang, "Enhanced electroluminescence of silicon-rich silicon nitride light-emitting devices by $\mathrm{NH}_{3}$ plasma and annealing treatment", Physica E 41, 920-922 (2009).

[12]. A. Morales, C. Domínguez, J. Barreto, M. Riera, M. Aceves, J. A. Luna, Z. Yu, R. Kiebach, “Spectroscopical analysis of luminescent silicon rich oxide films", Rev. Mex. Fis. S 53, 279-282 (2007).

[13]. A. Morales-Sánchez, J. Barreto, C. Domínguez, M. Aceves, Z. Yu, J. A. Luna-López, “Coulomb blockade effects in silicon nanoparticles embedded in thin silicon-rich oxide films", Nanotechnology 19, 165401 (2008).

[14]. M. Perálvarez, J. Barreto, J. Carreras, A. Morales, D. Navarro-Urrios, Y. Lebour, C. Domínguez, B. Garrido, "Si-nanocrystal-based LEDs fabricated by ion implantation and plasma-enhanced chemical vapor deposition", Nanotechnology 20, 405201 (2009).

[15]. http://www.srim.org/

[16]. A. Morales-Sánchez, J. Barreto, C. Domínguez, M. Aceves, J. A. Luna-López, "The mechanism of electrical annihilation of conductive paths and charge trapping in silicon-rich oxides", Nanotechnology 20, 45201 (2009).

[17]. A. A. G. Fernández, M. A. Mijares, A. M. Sánchez, K. M. Leyva, "Intense whole area electroluminescence from low pressure chemical vapor deposition-silicon-rich oxide based light emitting capacitors", J. Appl. Phys. 108, 043105 (2010).

[18]. A. Morales-Sánchez, J. Barreto, C. Domínguez-Horna, M. Aceves-Mijares, J. A. Luna-López, “Optical characterization of silicon rich oxide films", Sensor. Actuat. A - Phys. 142, 12-18 (2008).

\section{Introduction}

Despite the huge success of silicon in the electronics industry, it falls short of the efficient optical emission shown by direct band gap semiconductors. Since Canham first demonstrated light emission from silicon nanostructures back in 1990 [1], a lot of effort has been devoted to overcome the inherent limitations of bulk silicon as a light emitter.

In the last years, materials based on silicon nanoparticles embedded in silicon dioxide [2-7] and silicon nitride [8-11] have been shown to be Silicon nanoparticles embedded in a dielectric matrix can be fabricated by a number of techniques. Particularly interesting are low 
pressure chemical vapor deposition (LPCVD), plasma enhanced chemical vapor deposition (PECVD) and ion implantation, for these technologies are CMOS compatible and therefore make possible the monolithic integration of a silicon based light source in a conventional electronic circuit. These techniques allow us to obtain materials with similar electro-optical characteristics but with diverse electrical behaviors.

In order to analyze the effect that the fabrication technique has on the electrical behavior of the SRO materials, in the following sections we present the $\mathrm{C}-\mathrm{V}$ and $\mathrm{I}-\mathrm{V}$ characteristics of electroluminescent metaloxide-semiconductor (MOS) structures with silicon nanoparticles embedded in the dielectric layer [12], fabricated by LPCVD, PECVD and ion implantation. These are preliminary results of an in depth study of the mechanisms responsible for the electroluminescence in SRO materials.

\section{Experimental}

We have fabricated MOS structures as depicted in Fig. 1, where the active layer is formed by silicon rich silicon oxide instead of regular silicon dioxide. The active layer is grown/deposited on a 0.1-1.4 cm silicon substrate. Three sets of samples will be studied here, each with its active layer fabricated by one of the previously mentioned CMOS compatible techniques, namely LPCVD, PECVD and ion implantation.

The LPCVD and PECVD SRO layers were obtained adjusting the ratio between the precursor gases $\left(\left[\mathrm{SiH}_{4}\right] /\left[\mathrm{N}_{2} \mathrm{O}\right]\right)$. Samples with different ratios have been fabricated, thus leading to different silicon excesses in the active layers. More details on the preparation of the SRO samples have been published for LPCVD [13] and PECVD [7].

For the implanted samples, a $40 \mathrm{~nm}$ thick thermal silicon oxide is grown on the silicon substrate, followed by the deposit of a $30 \mathrm{~nm}$ thick silicon nitride layer used to control the silicon implantation process, which was removed after the annealing process.
Two silicon ion implantations were carried out [14] in each sample with varying doses (between $1.2 \times 10^{16}$ and $8.3 \times 10^{16} \mathrm{~cm}^{-2}$ ): the first one at $25 \mathrm{keV}$ and the second one at $50 \mathrm{keV}$. The implanted dose during the $50 \mathrm{keV}$ process is four times the implanted dose during the $25 \mathrm{keV}$ implantation. This ratio between the 25 and 50 $\mathrm{keV}$ implantations leads to a rather homogeneous distribution of the implanted silicon ions across the silicon oxide layer according to SRIM [15] simulations.

The experimentally obtained silicon excesses and thicknesses of all the samples can be found in Table I.

Once an SRO layer is obtained, a thermal annealing is carried out to induce the formation of silicon nanoparticles in the dielectric layer $[7,13,14]$. The selection of annealing temperatures and durations have been based on previous results $[7,13,14]$ and are referenced in Table I. In the case of the ion implanted samples, the silicon nitride layer used during the implantation process is removed after the annealing.

The device fabrication continues with the deposition and doping process of a $350 \mathrm{~nm} \mathrm{n-}$ type polysilicon layer followed by photolitography and etching to define the
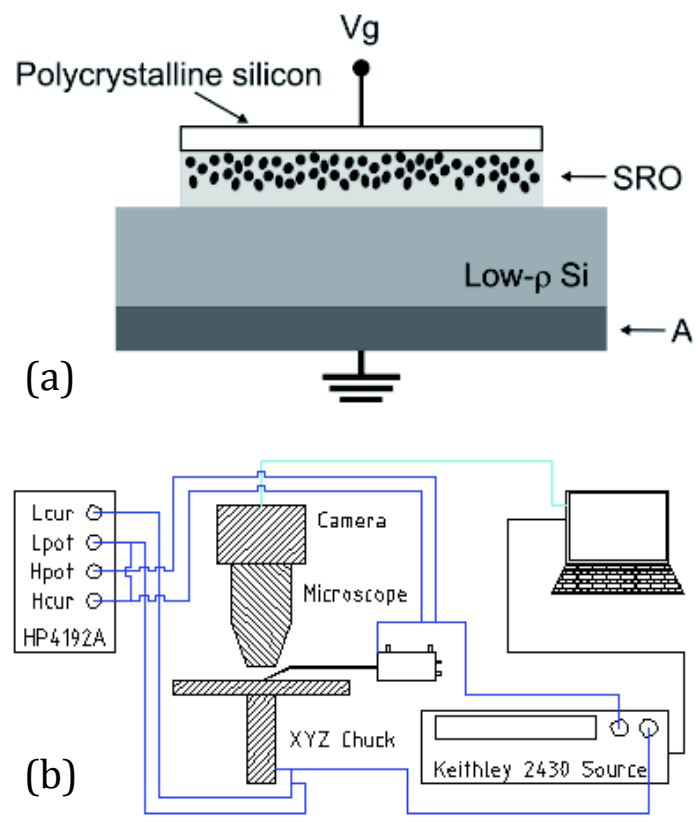

Fig. 1: (a) Vertical layout of the device and (b) measurement setup. 
Table I

Sample characteristics according to the fabrication technique.

\begin{tabular}{|c|c|c|c|}
\hline Technique & $\begin{array}{c}\text { Si. exc } \\
{[\%]}\end{array}$ & $\begin{array}{c}\text { Thickness } \\
{[\mathbf{n m}]}\end{array}$ & $\begin{array}{c}\text { Ann. temp. Ann. duration } \\
{\left[{ }^{\circ} \mathbf{C}\right]}\end{array}$ \\
\hline \multirow{3}{*}{ [min] }
\end{tabular}

transversal layout of the gate (squares of sides $500 \mu \mathrm{m}$ ). Next, an aluminum layer of $1000 \mathrm{~nm}$ is deposited followed by a second photolitography step and etching process to define the contact pads. Finally, the back contact is formed by sputtering of an aluminum layer followed by an annealing process.

The electrical measurements were performed in a Karl Süss probe station, with Süss Microtech PH120 probeheads and $7 \mu \mathrm{m}$ tungsten probes.

Current-voltage characteristics were obtained with a Keithley 2430 source-meter and capacitance-voltage characteristics were measured with an HP 4192A LF impedance analyzer.

The measurement protocol has been the same in all samples: firstly, capacitance-voltage characteristic is measured on as-obtained devices (those that have never been electrically stressed previously in any way). Secondly, current-voltage characteristics are measured. Finally the $\mathrm{C}-\mathrm{V}$ is again measured after the I-V stress.

Capacitance-voltage curves have been taken from inversion to accumulation and back to inversion with a voltage ramp of $0.1 \mathrm{~V} / \mathrm{s}$ and the $\mathrm{AC}$ component of the ramp at $100 \mathrm{KHz}$.

\section{Results}

Figures 2 to 5 show typical $\mathrm{C}-\mathrm{V}$ and $\mathrm{I}-\mathrm{V}$ behaviors of samples with different silicon excess and fabricated using different techniques.
For clarity, only the most representative samples have been plotted. In order to eliminate the dependence of the capacitance on the area and thickness of the device, the values have been multiplied by the thickness of the device and divided by its area and the permittivity of vacuum. Therefore, the values of the y-axis of the $\mathrm{C}-\mathrm{V}$ curves are adimensional and the saturation value in accumulation (negative voltages) tends to the relative permittivity of the medium.

The samples fabricated by LPCVD show two different behaviors: on as-obtained devices the $\mathrm{C}-\mathrm{V}$ curve is quite anomalous (red line, Fig. 2(a)). This corresponds to the high leakage current shown in Fig. 2(b) (red line). In this state, electroluminescence can be seen as bright spots on the surface of the device (Fig. 6(a)).

At some point during the electrical stress, the current drops to a low leakage situation [16]. At the same time the luminescent dots disappear [17]. Once in low leakage, the device remains in that state (see green line in Fig. 2(b), a second I$\mathrm{V}$ measurement). Moreover, if we measure capacitance again, the $\mathrm{C}-\mathrm{V}$ curve now resembles that of a regular MOS capacitance (see green line in Fig. 2(a)). While in low leakage, homogeneous luminescence in the whole area of the device can be detected from around $1.0 \mathrm{~A} \cdot \mathrm{m}^{-2}$. A picture of homogeneous emission is shown in Fig. 6(b) (the device in this picture corresponds to an Ion Implanted sample).

Figures 3(a) and 3(b) show the typical behavior of PECVD samples with different silicon 

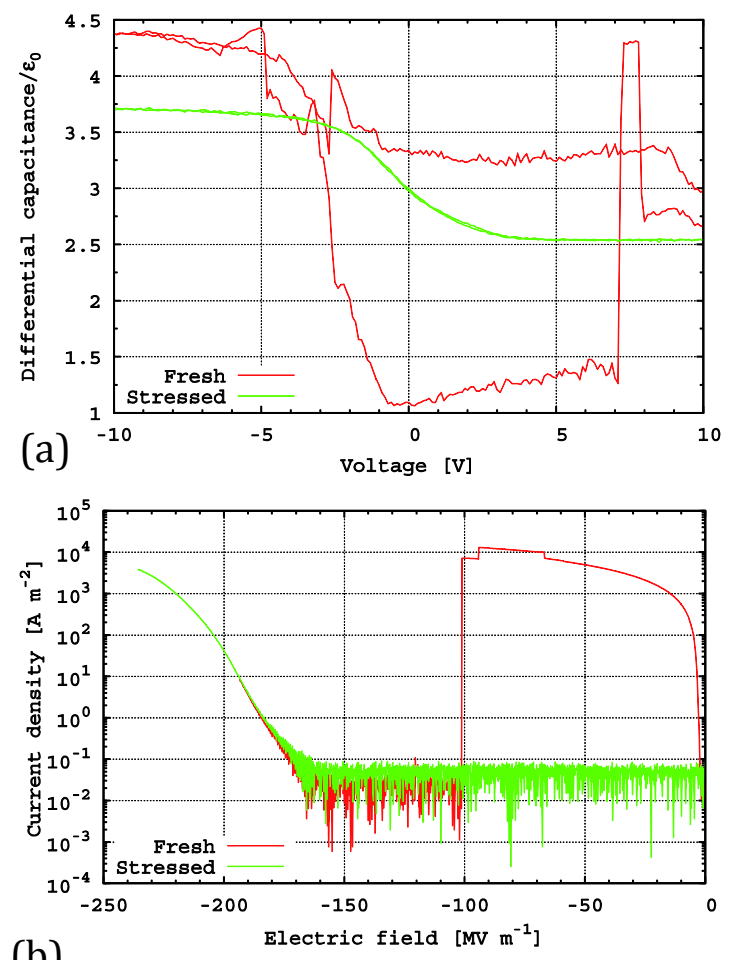

(b)

Fig. 2. Electrical characteristics obtained in LPCVD samples with $6 \%$ silicon excess. (a) $\mathrm{C}-\mathrm{V}$ characteristics; (b) I-V characteristics.

excess. The $\mathrm{C}-\mathrm{V}$ characteristics are quite similar to the expected from a MOS capacitance. Deep depletion can be observed in most samples and a displacement of the flat band voltage before and after the electrical stress can be seen for the lower silicon excesses. According to the hysteresis cycle, the amount of trapped charge and the oxide capacitance both increase along with silicon excess. The former is related to the increase of the density and size of the silicon nanoparticles [18]. From the latter we can infer an increase of the effective permittivity of the medium with the silicon excess[2], which is clear in the adimensional scale chosen for the $y$-axis of the $\mathrm{C}-\mathrm{V}$ plots.

As with the LPCVD samples, a regular $\mathrm{C}-\mathrm{V}$ curve is related to a low leakage current density (see Fig. 3(b)) and to homogeneous electroluminescence at around $1.0 \mathrm{~A} \cdot \mathrm{m}^{-2}$. No luminescent dots are observed in PECVD samples.

The current density increases with silicon excess. However, samples with higher silicon excess are also more prone to breakdown. As a
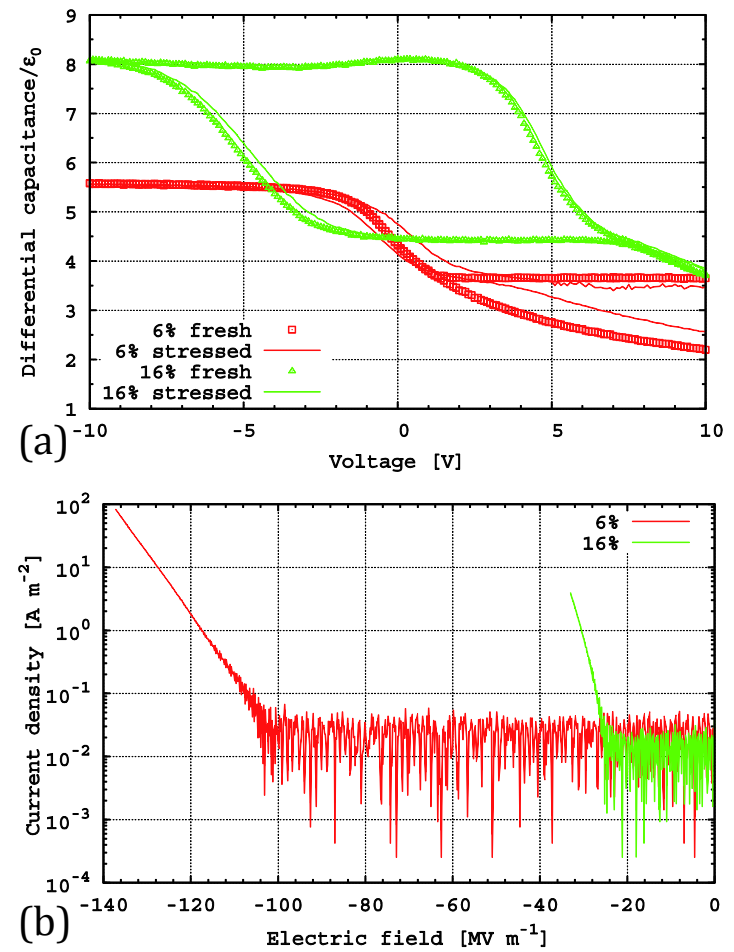

Fig. 3: Electrical behavior of PECVD samples with $6 \%$ and $16 \%$ silicon excess. (a) $\mathrm{C}-\mathrm{V}$ characteristics; (b) I-V characteristics.

result, luminescence appears at lower voltages but the maximum achievable current before breakdown is also lower.

Ion implanted samples show a slightly different $\mathrm{C}-\mathrm{V}$ behavior (Fig. 4(a)). According to the width of the hysteresis cycle, the amount of trapped charge decreases as the silicon excess is increased. This could be explained by considering the charge trapping as being affected by two different effects. One is how much charge can potentially be trapped in the layer (which would be related to the size and density of silicon nanoparticles) and the other is how easily can the layer be discharged. It seems that, in ion implanted samples, the increase in conductivity brought by the increase in silicon excess (and therefore the increase in the discharging ability) has a larger effect than the increase in the size and density of the silicon nanoparticles (and therefore the increase in the charge trapping ability).

There is also very little change in the $\mathrm{C}-\mathrm{V}$ curves before and after the electrical stress. As with the PECVD samples, the relative permittivity of the medium increases with 

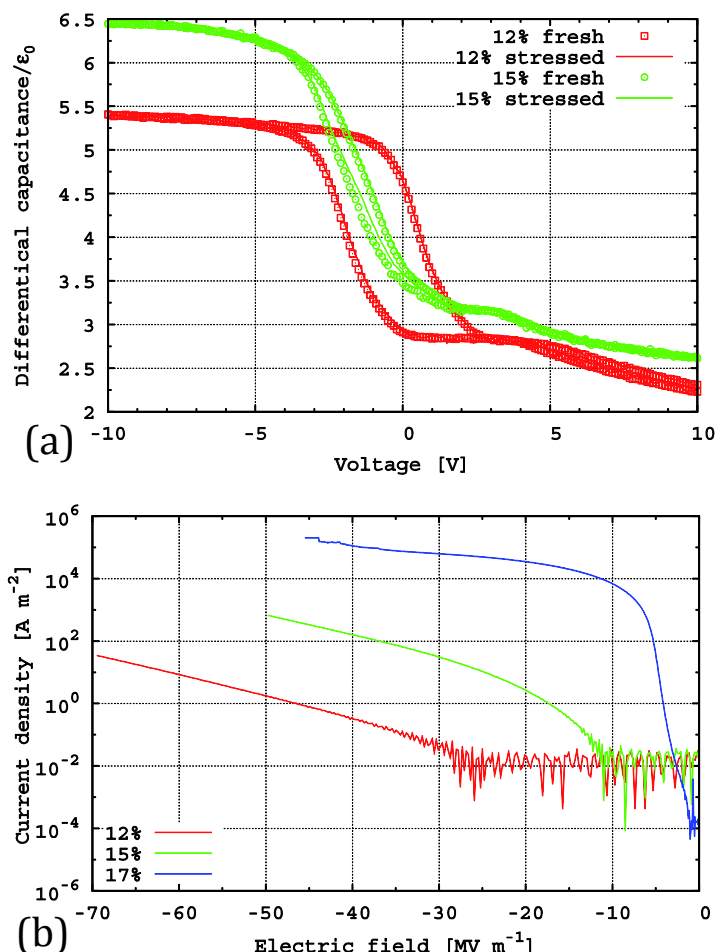

Fig. 4. Electrical characteristics of implanted samples with $12 \%$ and $15 \%$ silicon excess. (a) C-V characteristics; (b) I-V characteristics.

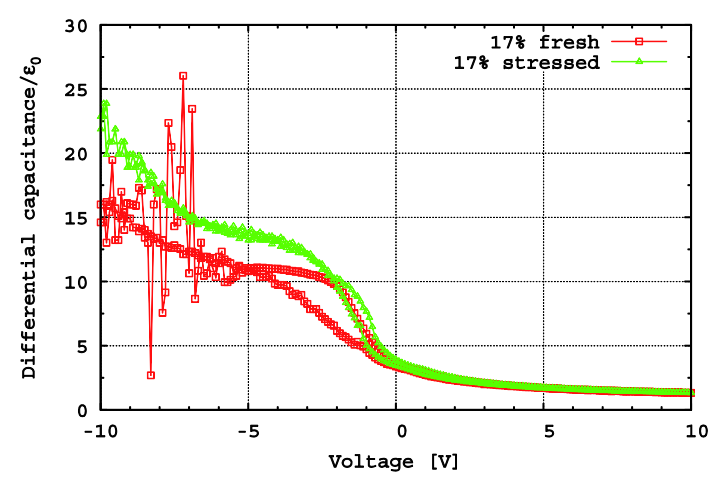

Fig. 5. Anomalous $\mathrm{C}-\mathrm{V}$ curve of the implanted sample with $17 \%$ silicon excess

silicon excess, although there is little difference between the samples with $14 \%$ and $15 \%$ silicon excess. The samples with silicon excesses from $12 \%$ to $15 \%$ present homogeneous electroluminescence in the whole area from 1.0 A. $\mathrm{m}^{-2}$ (Fig. 6(b)).

The sample with $17 \%$ silicon excess presents an unstable $\mathrm{C}-\mathrm{V}$ curve (Fig. 5) as well as high leakage current (Fig. 4(b), blue line). Similarly to what happened in the high leakage state in the LPCVD samples, no homogeneous electroluminescence is observed in this sample. Instead, only luminescent dots can be seen.
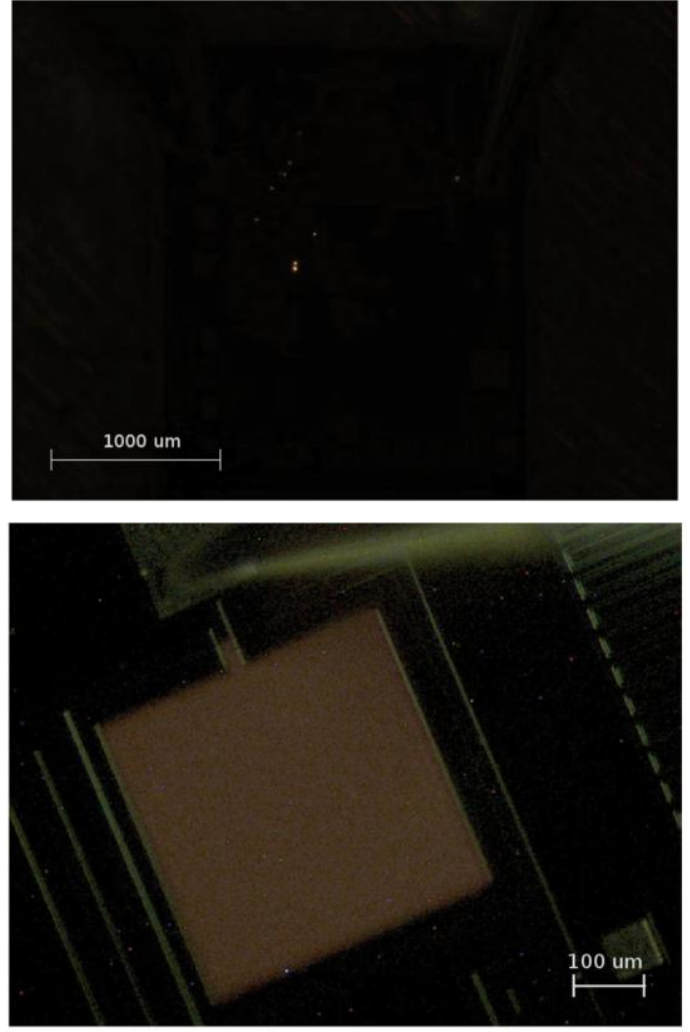

Fig. 6. Two kinds of luminescent behavior. The dots are related to a high conduction state through the luminescent points, whereas the homogeneous luminescence is related to an also homogeneous low conduction state. (a) Luminescent dots (LPCVD); (b) Homogeneous luminescence (Ion Implantation).

In the I-V curves of the implanted samples it is also observed that the current density increases with silicon excess. The considerations about breakdown that we have discussed for the PECVD samples also apply here, although the differences are much less apparent, most likely due to the relatively small difference in silicon excess between samples.

\section{Conclusions}

We have shown the $\mathrm{C}-\mathrm{V}$ and $\mathrm{I}-\mathrm{V}$ behavior of MOS structures featuring silicon nanoparticles embedded in its dielectric matrix.

We have seen that there are two possible conduction regimes, one with low leakage and one with high leakage. The high leakage regime seems to be related to the formation of a finite number of conductive paths across the dielectric matrix. In this situation, almost all the current flows through those conductive paths. Therefore, 
the conduction through the active layer is not uniform and light is only observed in points corresponding to the places where the conduction takes place.

On the other hand, in the low leakage regime there are no such conductive paths and the current flows uniformly through the whole area of the layer. Accordingly, light emission also occurs uniformly in the whole area of the device.

The studied PECVD samples do not show a high leakage regime, whereas for implanted samples only the highest silicon excess shows such regime. The LPCVD sample presents both kinds of leakage: as obtained devices show high leakage, which turns into low leakage after the application of an electrical stress. This behavior has been attributed to the annihilation of the preferential conductive paths [16].
It has also been shown that the low leakage regime is related to a regular MOS-like $\mathrm{C}-\mathrm{V}$ behavior, whereas samples in high leakage regime show anomalous $\mathrm{C}-\mathrm{V}$, most likely related to the instability of the conductive paths.

A remarkable fact is that all the devices have a threshold current density of $1.0 \mathrm{~A} \cdot \mathrm{m}^{-2}$ before the emission starts, independently of the fabrication technique. This threshold is achieved at different electric fields depending on the silicon excess and the fabrication technique.

\section{Acknowledgements}

A. González acknowledge the grant No. 213571 received from the CONACyT of México. 